\title{
ANTIBACTERIAL EFFECT OF CALCIUM HYDROXIDE IN DIFFERENT VEHICLES
}

K. R. Hari, M. R. Ganesh,

1. Professor, M.D. S. Department of Oral Surgery, Adhiparashakti Dental College, Chennai.

2. Reader, M.D. S. Department of Endodontics, Adhiparashakti Dental College, Chennai.

\section{CORRESPONDING AUTHOR}

Dr. M. R. Ganesh

C. S.T, back of G. P. 0

Mumbai-1, Maharashtra, India,

E-mail: watvrukshpippal909@gmail.com,

Ph: 00918600117037.

ABSTRACT: AIM: This study evaluated the antibacterial effect of calcium hydroxide in different vehicles in an in vitro model. MATERIAL AND METHODS: Calcium hydroxide paste prepared with two conventionally used vehicles namely, camphorated monochlophenol, distilled water and also propylene glycol. The antibacterial activity of these paste were tested against five micro- organisms that can commonly occur in the infected root canals. RESULTS AND CONCLUSIONS: The results of the study indicate that a paste of calcium hydroxide made with propylene glycol exerts significant antibacterial action. Hence, it can be recommended for use as an intracanal medicament in preference to a paste prepared with a tissue toxic phenolic compound like camphorated mono chlorophenol.

KEY WORDS: Calcium hydroxide, camphorated monochlorophenol, propylene glycol.

INTRODUCTION: The endodontist today, concentrates more upon instrumentation, irrigation thorough cleaning and shaping of the root canal for elimination of responsible microbial flora from the root canal. [12], [15],[17],[21]

With due respect to all norms laid down in the field of endodontics, the review of literature is suggestive that establishing the complete debridement and disinfection of the root canal contributes to maximum therapeutic success.

In routine clinical practice, disinfection of the root canal is established by comprehensive effect of biomechanical preparation, use of intra canal irrigation and intra canal medicament. [1], [23],[14]

Calcium hydroxide is known to endodontists for its application from many years since its introduction by Herman in 1930.

In present day, it has become more popular as intra canal medicament. Its bactericidal or bacteriostatic action is related to its dissociation into calcium and hydroxyl ions thereby creating alkaline environment in its vicinity, not allowing the growth of acidophilic microorganisms. In addition to this it also inhibits the enzymatic activity that is essential for microbial growth. ${ }^{[8],[9]}$

To facilitate its application in the field of endodontics, it is generally mixed with vehicles like camphorated mono chloro phenol, distilled water, glycerin and propylene glycol. Various studies are carried out to identify or to understand the most suitable vehicle and also to find out the effect of vehicle on the action of the calcium hydroxide as an intra canal medicament. Observations followed to such study suggest that calcium hydroxide when mixed with water as 
a vehicle promotes the dissociation and initiates the meditational action of the calcium hydroxide.

Calcium hydroxide when mixed with camphorated mono chlorophenol has dual effect vehicle as well as additional antibacterial action.

Calcium hydroxide when mixed with propylene glycol also has dual effect like camphorated mono chlorophenol. However, it exhibits no tissue irritating effect and being alcoholic in nature, it remains in paste form for longer time.

However there are very few study related to propylene glycol that serves as better vehicle when mixed with calcium hydroxide to form a paste for the purpose of intracanal medicament.

It was purposed to comparatively evaluate the antibacterial effect of calcium hydroxide when mixed with distilled water, camphorated mono chlorophenol and propylene glycol.

MATERIAL AND METHODS: Infected Root Canal Flora Commonly Consist of gram positive cocci like streptococci, staphylococci, gram positive bacilli like acidophillus, lactobacilus, actinomycotis fungi like candida albicans, gram negative organism like E-coli, pseudomonas.

More common and more resistant organisms in root canals are streptococci and E. Faecalis respectively.

Considering infected microbial flora of infected root canal it was purposed to obtained the five micro organisms samples from the stock culture of the department of microbiology, grand medical college, Sir. J.J. Group of Hospital Mumbai, namely

1) H. Streptococcus,

2) Enterococcus Faecalis

3) Staphylococcus aureus

4) Pseudomonas aeruginosa

5) Candida albicans

Calcium hydroxide powder was pre-weighed (1 gm.) and stored in autoclaved vials. Calcium hydroxide paste preparations used in the study are:

1. Calcium Hydroxide and distilled water

2. Calcium Hydroxide and propylene glycol $90 \%$

3. Calcium Hydroxide and propylene glycol $100 \%$

4. Calcium Hydroxide and camphorated monochlorophenol

The ratio used in this study to prepare paste was 1:1 that $1 \mathrm{gm}$ calcium hydroxide powder with $1 \mathrm{CC}$ of appropriate liquid. Sterile instrument were used for each preparation. Such prepared sample pastes were divided in to 3 different groups namely

\section{Group 1}

Calcium hydroxide \& distill water (control group), 1gm of calcium hydroxide powder mixed with $1 \mathrm{cc}$ of distilled water.

\section{Group 2}

Calcium hydroxide \& propylene glycol (Experimental group). Group two was experimental group, which was further subdivided in to two sub groups 
Group 2 A: - Calcium hydroxide \& propylene glycol 100\%, 1gm. of calcium hydroxide powder mixed with 1 cc $100 \%$ propylene glycol.

Group 2 B: - Calcium hydroxide \& propylene glycol 90\%, 1gm. of calcium hydroxide powder mixed with 1 cc $100 \%$ propylene glycol.

Group 3 :- Calcium hydroxide \& Camphorated monochlorophenol 1gm. of calcium hydroxide powder mixed with $1 \mathrm{cc}$ camphorated monochlorophenol.

Solid media were used for propagating the organisms, which were tested for sensitivity by ditch plate. Glucose broth, peptone water, nutrient broth for aerobes and Sabouraud's dextrose broth for fungal organisms.

Four wells of $5 \mathrm{~mm}$ diameter were cut on each solid media using a sterile metallic punch fitted with bulbed teat. A standard loop with an internal diameter of $4 \mathrm{~mm}$, which could deliver $0.01 \mathrm{ml}$ of the suspension of culture of test organism, then spread a cross the plate using sterile cotton swab.

The wells were then filled with the 4 different preparation of calcium hydroxide and the culture plates were then incubated.

The plates of aerobic organisms were incubated aerobically at $37 \mathrm{oC}$ and the zone of inhibition measured after 24, 48 and 72 hours accordingly.

The antibacterial sensitivity pattern represented as the zone of inhibition at its maximum diameter was measured around each well using a caliper and results observed were tabulated.

RESULTS: In the present study, in group one (1) was control group where one gram of calcium hydroxide powder was mixed with one cc of distilled water to form a paste. The result showed that calcium hydroxide distilled water paste produced zone of inhibition that was significantly smaller than other groups, only Candida albicans exhibited a large zone of inhibition. It requires more incubation period. It is, well-established fact that calcium hydroxide has antibacterial property. When calcium hydroxide paste prepared with distilled water, water acts as a vehicle only. It does not possess antibacterial property.

In-group 2 where 1 gram of calcium hydroxide powder was mixed with $1 \mathrm{cc}$ of propylene glycol to form paste

Group 2 further divided into two sub groups

Group 2A, where $1 \mathrm{gm}$. of calcium hydroxide powder mixed with $1 \mathrm{cc}$ of $100 \%$ of propylene glycol.

Group $2 \mathrm{~B}$, where $1 \mathrm{gm}$. of calcium hydroxide powder mixed with 1cc of $90 \%$ propylene glycol.

It was observed in the present study that among all the calcium hydroxide paste, only calcium hydroxide propylene glycol paste remain in the paste form for longer period of time which indicate his good handling qualities and exerts significant antibacterial action. The average diameter of the zone of inhibition for both groups calcium hydroxide and $90 \%$ propylene glycol and calcium hydroxide and $100 \%$ propylene glycol combination were similar.

In group-III, where $1 \mathrm{gm}$ of calcium hydroxide powder was mixed with $1 \mathrm{cc}$ of camphorated Mono Chloro Phenol (CMCP) to form a paste. These pastes gave the best results as a maximum zone of inhibition.

Different preparation of calcium hydroxide used in this study produced some degree of inhibition of growth of the test organisms including enterococci. The average diameter of the zones of inhibition for aerobic organisms is shown in table 1. The inhibition of growth was 
exhibited as clear zone, around the well. It had the appearance of a fresh agar surface and was smooth and clear. Besides the zone of inhibition, there was another distinct zone, which was white in color. The zones sometimes extended even beyond the inhibition zone and appeared to be the diffusion of Calcium hydroxide through the agar medium. There was also a slight brownish discoloration around the immediate periphery of the phosphates due to the increased alkaline $\mathrm{PH}$ in this area of the agar.

Among the various preparation of Calcium hydroxide, Calcium hydroxide camphorated monochlorophenol combination demonstrated the highest degree of growth inhibition; the entire organisms exhibited a fairly high sensitive to this paste.

The average diameter of the zone of inhibition for both Experimental group Calcium hydroxide with $90 \%$ propylene glycol \& Calcium hydroxide with $100 \%$ propylene glycol combinations were equal. In case of Calcium hydroxide and distilled water paste, as a control group, only Candida albicans exhibited a large zone of inhibition.

There was distinct growth inhibition observed with enterococcus on 3 days of incubation with Calcium hydroxide camphorated monochlorophenol paste. Candida albicans showed no growth on the first day of incubation in all specimens. It required more incubation period.

\section{Antibacterial Activity of Calcium Hydroxide with Various Vehicles}

\begin{tabular}{|c|c|c|c|c|c|c|}
\hline \multirow{2}{*}{$\begin{array}{l}\text { Sr.N } \\
\text { o. }\end{array}$} & \multirow{2}{*}{$\begin{array}{l}\text { ORGANISM } \\
\text { (Fungal and Aerobic) }\end{array}$} & \multicolumn{5}{|c|}{ Zone Of Inhibition In MM } \\
\hline & & $\begin{array}{l}\text { Days Of } \\
\text { Incubation }\end{array}$ & $\begin{array}{l}\mathrm{CH}+ \\
\text { Distilled } \\
\text { Water }\end{array}$ & $\begin{array}{l}\mathrm{CH}+ \\
90 \% \\
\mathrm{PG}\end{array}$ & $\begin{array}{l}\mathrm{CH}+ \\
100 \% \\
\mathrm{PG}\end{array}$ & $\begin{array}{l}\mathrm{CH}+ \\
\mathrm{CMCP}\end{array}$ \\
\hline & \multirow[t]{3}{*}{ Candida Albicans } & \multicolumn{5}{|c|}{$1^{\text {st }}$ (no growth) Candida required more incubation period } \\
\hline & & $2^{\text {nd }}$ & 22 & 24 & 24 & 34 \\
\hline & & $3^{\text {rd }}$ & 22 & 25 & 24 & 36 \\
\hline & \multirow[t]{3}{*}{ Haemolytic treptococcus } & 1 st & 15 & 18 & 18 & 30 \\
\hline & & $2^{\text {nd }}$ & 16 & 19 & 19 & 32 \\
\hline & & $3^{\text {rd }}$ & 16 & 19 & 19 & 32 \\
\hline & \multirow[t]{3}{*}{ Enterococcus } & $1 \mathrm{st}$ & $\mathrm{R}$ & $\mathrm{R}$ & $\mathrm{R}$ & 16 \\
\hline & & $2^{\text {nd }}$ & $\mathrm{R}$ & $\mathrm{R}$ & $\mathrm{R}$ & 16 \\
\hline & & $3^{\text {rd }}$ & $\mathrm{R}$ & $\mathrm{R}$ & $\mathrm{R}$ & 16 \\
\hline & \multirow{3}{*}{$\begin{array}{l}\text { Staphylococcus } \\
\text { Aureus }\end{array}$} & 1st & 13 & 13 & 14 & 30 \\
\hline & & $2^{\text {nd }}$ & 13 & 14 & 14 & 30 \\
\hline & & $3^{\text {rd }}$ & 13.5 & 16 & 16 & 32 \\
\hline & \multirow[t]{3}{*}{ Pseudomonas Aeroginosa } & $1 \mathrm{st}$ & 12 & 12 & 14 & 26 \\
\hline & & $2^{\text {nd }}$ & 12 & \begin{tabular}{|l}
12 \\
\end{tabular} & 14 & 29 \\
\hline & & $3^{\text {rd }}$ & 12 & 12 & 14 & 29 \\
\hline
\end{tabular}

Journal of Evolution of Medical and Dental Sciences/Volume1/Issue5/November-2012Page-741 
DISCUSSION: It is, well-known fact that success in endodontic therapy depends upon adhering strictly to various norms laid down namely diagnostic phase, preparatory phase and phase of obturation. [7], [13],[24]

The endodontist today concentrates more upon instrumentation, irrigation thorough cleaning and shaping of the root canal for elimination of responsible microbial flora from the infected root canal.

Calcium hydroxide is effective in eliminating microorganisms from the root canal space. [11]

Calcium hydroxide has bactericidal effect and its capacity to neutralize the bacterial endotoxins. ${ }^{[20]}$

In the present study, in group one as control group where one gram of calcium hydroxide powder was mixed with one cc of distilled water to form a paste. The result showed that calcium hydroxide distilled water paste produced zone of inhibition that was significantly smaller than other groups, only Candida albicans exhibited a large zone of inhibition. It requires more incubation period. It is, well-established fact that calcium hydroxide has antibacterial property. When calcium hydroxide paste prepared with distilled water, water acts as a vehicle only. It does not possess antibacterial property. [4], [6],[10],[18]

Paul K. evaluated the antibacterial efficacy of calcium hydroxide with different vehicles like distilled water, camphorated monochloro phenol, and propylene glycol. The results showed that calcium hydroxide paste with camphorated monochloro phenol, and propylene glycol was more effective as compare to the calcium hydroxide distilled water paste. [16]

Antony B studied the antibacterial property of calcium hydroxide with four different vehicles like distilled water, glycerin, camphorated monochloro phenol and propylene glycol. The result of the study showed that all the different preparation of calcium hydroxide paste with glycerin, camphorated monochloro phenol and propylene glycol was more effective in controlling the microorganisms as compared to calcium hydroxide distilled water paste. [3]

In-group II where 1 gram of calcium hydroxide powder was mixed with $1 \mathrm{cc}$ of propylene glycol to form paste

Group II further divided into two sub groups

Group II A, where 1gm. of Calcium Hydroxide powder mixed with $1 \mathrm{cc}$ of $100 \%$ of propylene glycol.

Group II B, where $1 \mathrm{gm}$. Of Calcium Hydroxide powder mixed with 1cc of 90\% propylene glycol.

It was observed in the present study that among all the Calcium Hydroxide paste, only Calcium Hydroxide propylene glycol paste remain in the paste form for longer period of time which indicate his good handling qualities and exerts significant antibacterial action. The average diameter of the zone of inhibition for both groups Calcium Hydroxide $+90 \%$ propylene glycol and Calcium Hydroxide $+100 \%$ propylene glycol combination were similar.

Antibacterial property of Calcium Hydroxide in different vehicles an in vitro evaluated by the Paulk $100 \%$ and $90 \%$ propylene glycol as vehicle used to make the paste with Calcium Hydroxide. The result of the study showed that the paste of the Calcium Hydroxide made with propylene glycol exert significant antibacterial action and it can be considered for use as an intra canal medicament in preference to the paste prepared with tissue irritating phenolic compound like camphorated monochloro phenol. [2]

Propylene glycol which was suggested by Laws in (1962) has been evaluated for its possible use as a vehicle in the field of endodontics. ${ }^{[5]}$ It permits the release of Calcium and 
Hydroxyl ions essential for the therapeutic action. It has been found to be antibacterial, not irritating to the periapical tissue. Both 90 and 100\% propylene glycol Calcium Hydroxide paste showed antibacterial properties, which might be due to the slow diffusion of the Calcium and Hydroxyl ions as compared to the paste prepared with distilled water. The addition of $10 \%$ water was done in group II B with view of facilitating immediate ionization of the Calcium Hydroxide which is necessary for its action. The addition of water did not facilitate the enhancement of antibacterial action.

In group-III, where 1gm of calcium hydroxide powder was mixed with 1cc of camphorated Mono Chloro Phenol (CMCP) to form a paste. These pastes gave the best results as a maximum zone of inhibition. Its clinical use is objectionable due to its tissue irritating property. The size of the zone of bacterial inhibition does not necessarily reflect the strength of antibacterial agent. The zone size may be influenced by the molecular size of the chemical and its diffusion. An agent that diffuses more easily will exhibit a large zone and camphorated monochloro phenol has been shown to diffuse readily through blood agar medium. Since disinfection of the root canal is established by comprehensive effect of Biomechanical Preparation, irrigation and Intra canal Medicament.

The loss of long-term activity can be attributed to the insoluble weak salt formed in combination with the hydroxyl ions dissociation, thus affecting the biological activity of calcium hydroxide. [19]

Calcium hydroxide Camphorated Mono Chloro Phenol paste has a tendency to become sticky and set fast.

Besides, camphorated monochloro phenol in volatile liquid can be lost from the paste rapidly.

The toxicity of phenol, camphorated phenol and camphorate mono chloro phenol was evaluated by Saekonto and confirmed the cytotoxicity of these antiseptic. They are irritants to the Periapical tissue. [22]

Even camphorated monochloro phenol alone or with calcium hydroxide is effective more than other two pastes but it is not prefer as it causes irritation to the periapical tissue.

Considering the result of the study and revive of literature, it can be said that: -

1. Both $90 \%$ and $100 \%$ propylene glycol paste of calcium hydroxide are bactericidal agents against microorganisms commonly found in infected root canals.

2. Camphorated monochlorophenol calcium hydroxide paste is most effective bactericidal agent as compared to other two pastes.

3. The routine use of propylene glycol in place of camphorated monochlorophenol can be recommended as vehicle for calcium hydroxide in view of its efficacy and non-irritating property to the periapical tissues.

\section{CONCLUSIONS:}

The results of this study are suggestive of: -

1. Both $90 \%$ and $100 \%$ propylene glycol paste of calcium hydroxide are bactericidal agents against microorganisms commonly found in root canals.

2. Calcium hydroxide camphorated monochlorophenol paste is more effective bactericidal agent as compared to the other two pastes. 
3. The routine use of propylene glycol in place of camphorated monochlorophenol can be recommended as vehicle for calcium hydroxide in view of its efficacy and better handling properties.

4. The diffusion of calcium hydroxide propylene glycol paste along with tissue irritation and its efficacy in vivo, require further investigation.

\section{REFERENCES:}

1. Amanda Law, An evidenced based analysis of the anti bacterial effectiveness of intracanal medicaments. JOE,2004;30; 689-93.

2. Angky S. Shonei K., Shiro M., Kerichi O, Hideaki O. Toxicity of camphorated phenol and camphorated parachlorophenol in dental pulp cell culture. J. Endod 1996; 6 : 284-86.

3. Antony B, Comparitive evaluation of antibacterial efficacy of calcium hydroxide with four different vehicles. Ind JE 1997 : 9; 50 -55.

4. Behnen, Antimicrobial activity of several calcium hydroxide preparation in root canal dentin. J Endod 2000;27(12):765-67.

5. Bhat KS, Walvekar SV. Evalution of bactericidal property of propylene glycol for its possible use in endodontics. Arogya J. Health Sci 1975; 1:54-0.

6. Bystrom, The antimicrobial effect of camphorated monochlorophenol, camphorated phenol and calcium hydroxide in the treatment of infected root canals. Endod Dent Traumatol1985;1:170-75

7. Cohen, Cleaning and shaping of root canal,Textbook of Pathways of pulp.

8. Estrela C. Control of micro-organisms invitro by calcium hydroxide paste. Int Endodon J 2001;34:341-345.

9. Estrela $C$, In vitro determination of direct antimicrobial effect of calcium hydroxide JOE,1998;24; 15-17.

10. Estrela C, Mechanism of action of calcium and hydroxyl ions of calcium hydroxide on tissue and bacteria. Brazil Dent J. 1995;6:85-19.

11. Evans M, Sundqvist G, Mechanism in the resistance of E. faecalis to calcium hydroxide Int Endodont J, 2002;35:221-8.

12. Gianuck G, Shaping and cleaning the root canal system SEM study evaluation of new instrumentation and irrigation technique. JOE 1999;25; 800-03.

13. Grossman LI, Endodontic practice. 10th Ed. Philadelphia Lea and Febiger 1982;279(Ingle).

14. Gomes, In vitro antimicrobial activity of several concentrations of sodium hypochlorite and chlorhexidine gluconate in the elimination of E. faecalis. JOE 2000;34; $424-28$.

15. Keniolime, Chemomechanical reduction of the bacterial population in root canal after instrumentation and irrigation. JOE 2000.

16. Paul K, Antibacterial property of calcium hydroxide using different vehicles. Ind EJ $1997 ; 9 ; 43-49$.

17. Peters LB, Effect of instrumentation, irrigation and dressing with calcium hydroxide on infection in pulpless teeth with periapical lesion. Int Endod 2002;35:13-21.

18. Roy HS, Grossman LI. Evaluation of the antimicrobial potential of calcium hydroxide as an intracanal medicament J. Endod 1983; $9: 372-74$.

19. Safavi K, Influence of mixing vehicles on dissociation of calcium hydroxide in solution. J Endodon 2000;26:649-51. 
20. Safavi and Nichols, Alteration of biological properties of bacterial lipopolysaccharides by calcium hydroxide treatment. J Endodon 1994;20:127-29.

21. Siqueira JF, Mechanical reduction of bacterial population in the root canal by in strumentation technique. JOE 1999;25; 332-35.

22. Soekanto, Toxicity of camphorated phenol and camphorated monochlorophenol in dental pulp cell culture. JOE 1996;22; 284-86.

23. Tu Chawchany, Effect of camphorated monochlorophenol on human periodontal cells in vitro. JOE 1999;25.

24. Wein, Basis of successful endodontic therapy, Textbook of endodontics. $1996 ; 05 ; 01$ 27. 